\title{
Encoding Table Constraints in CLP(FD) Based on Pair-wise AC
}

\author{
Neng-Fa Zhou \\ CUNY Brooklyn College \& Graduate Center \\ zhou@sci.brooklyn.cuny.edu
}

\begin{abstract}
We present an implementation of table constraints in CLP(FD). For binary constraints, the supports of each value are represented as a finite-domain variable, and action rules are used to propagate value exclusions. The bit-vector representation of finite domains facilitates constant-time removal of unsupported values. For n-ary constraints, we propose pair-wise arc consistency (AC), which ensures that each value has a support in the domain of every related variable. Pair-wise AC does not require introducing new problem variables as done in binarization methods and allows for compact representation of constraints. Nevertheless, pair-wise AC is weaker than general arc consistency (GAC) in terms of pruning power and requires a final check when a constraint becomes ground. To remedy this weakness, we propose adopting early checks when constraints are sufficiently instantiated. Our experimentation shows that pair-wise AC with early checking is as effective as GAC for positive constraints.
\end{abstract}

\section{Introduction}

A table constraint, or extensional constraint, over a tuple of variables specifies a set of tuples that are allowed (called positive) or disallowed (called negative) for the variables. Recently there has been a growing interest in this format of constraints. This format is well suited to problems where relations are more easily given in extension than in intension such as configuration problems involving datasets (e.g., crossword puzzles). Another reason for the popularity of this format is that certain intensional constraints, especially nonlinear and global constraints, can be more cheaply maintained when tabulated. The table format has been used in the CSP solver competitions and a good collection of problem instances are available. Arc consistency has been generalized for table constraints $[5,12]$ (called $G A C$ ) and several data structures have been proposed for maintaining GAC for table constraints $[2,3,6,7,9,10]$.

No previous work has been reported on introducing table constraints into CLP(FD). Because of the lack of sophisticated data structures such as multidimensional arrays and the necessity of manipulation of tagged data in CLP(FD), an efficient data structure designed for a low-level language may not be suited 
to $\mathrm{CLP}(\mathrm{FD})$. In this paper, we propose an encoding for table constraints in B-Prolog, a CLP(FD) system.

For a binary table constraint, the supports of each value are represented as a finite-domain variable. When either variable in the constraint is bound to a value, the other variable is unified with the finite-domain variable that represents the supports of the value. Whenever a value is excluded from the domain of a variable in the constraint, the supports of the value in the domain of the other variable are examined and those values that are no longer supported are excluded. As bit vectors are used to represent finite domains, the basic operations required in propagation can be performed efficiently [11].

For an n-ary table constraint, we propose pair-wise arc consistency (AC), which ensures that each value has a support in the domain of every related variable. As for binary constraints, supports of each value are also represented as a finite-domain variable. One of the advantages of pair-wise $\mathrm{AC}$ is that it, unlike binarization methods [1], does not introduce new problem variables. The newly introduced finite-domain variables are solely used as bit vectors to represent supports of values. Since supports are not updated during search, no events can occur in these new domain variables. This representation fits CLP(FD) since bits are not tagged individually. Another advantage of pair-wise $\mathrm{AC}$ is that constraints can be represented very compactly. Let $n$ be the arity of an n-ary contraint and $d$ be the size of the maximum domain. Supports of values can be represented with $O\left(n^{2} \times d^{2}\right)$ space.

Nevertheless, pair-wise AC is weaker than GAC in terms of pruning power because, understandably, it is impossible to use $O\left(n^{2} \times d^{2}\right)$ space to represent as many as $d^{n}$ tuples. To remedy this weakness, we propose adopting early checks to enforce GAC when constraints are sufficiently instantiated. Early checking extends forward checking [8] because the number of variables contained in a constraint can be more than one when the constraint is checked.

For each variable $X$ in a table constraint, a propagator is used to watch the ins $(X)$ event which is posted when $X$ is instantiated, and another propagator is used to respond to the dom_any $(X, E)$ event which is posted whenever any element $E$ is excluded from the domain of $X$. Propagators are described using action rules [16]. Our implementation propagates values like the AC-4 algorithm [13], and hence can be classified as fine-grained.

The contribution of this paper is twofold. First, this paper presents an encoding for table constraints which is suited to any CLP(FD) system that represents finite-domains as bit vectors and handles domain value exclusions as events. Second, this paper proposes pair-wise AC, which is a natural extension of AC but has never received much attention before, and proposes to remedy the weakness of pair-wise AC with early checking. We experimented with two different settings for early checking and our experimental results showed that pair-wise AC with early checking is as effective as GAC. 
This paper is organized as follows: Section 2 overviews table constraints, consistency algorithms, CLP(FD), and action rules; Section 3 describes an encoding for binary constraints and gives the propagators as action rules; Section 4 gives the propagators for maintaining pair-wise AC; Section 5 proposes several improvements on the propagators; Section 6 describes early checking; Section 7 presents the experimental results; Section 8 discusses the related and future work.

\section{Preliminaries}

\subsection{Table constraints and consistency}

A table constraint is either positive or negative. A positive constraint takes the form $X$ in $R$ and a negative constraint takes the form $X$ notin $R$ where $X$ is a tuple of variables $\left(X_{1}, \ldots, X_{n}\right)$ and $R$ is a table defined as a list of tuples of integers where each tuple takes the form $\left(a_{1}, \ldots, a_{n}\right)$. In order to allow multiple constraints to share a table, we allow $X$ to be a list of tuples of variables. In theory, a negative constraint can always be represented as a positive constraint by complementing the table, but in practice this is not always viable since the resulting table can be prohibitively large.

A table constraint is said to be binary if each tuple has only two components, and n-ary if each tuple has more than two components. A table constraint degenerates into a domain constraint in CLP(FD) if each tuple has only one component.

Let $\left(X_{1}, X_{2}\right)$ in $R$ be a binary table constraint. A value $x_{1}$ in the domain of $X_{1}$ is said to be supported in the constraint if there exists a value $x_{2}$ in the domain of $X_{2}$ such that $\left(x_{1}, x_{2}\right)$ is included in $R$. The constraint is said to be $A C$ (arc consistent) on $X_{1}$ if every value in the domain of $X_{1}$ is supported. The constraint is said to be $A C$ if it is $\mathrm{AC}$ on both $X_{1}$ and $X_{2}$.

Let $\left(X_{1}, \ldots, X_{n}\right)$ in $R$ be an n-ary table constraint. Let $R_{i j}$ denote the projection of the table $R$ over the $i$ th and $j$ th columns $(i<j)$. The binary projection of the constraint over $X_{i}$ and $X_{j}(i<j)$ is the binary constraint $\left(X_{i}, X_{j}\right)$ in $R_{i j}$. The n-ary constraint is said to be pair-wise $A C$ if all of its binary projections are AC.

Consider the n-ary constraint $\left(X_{1}, \ldots, X_{n}\right)$ in $R$ again. A value $x_{i}$ in the domain of variable $X_{i}$ is gac-supported in the constraint if there exists a tuple in $R$ whose $i$ th component is equal to $x_{i}$. The constraint is said to be $G A C$ if every value in the domain of every variable is gac-supported. This condition can be given more formally as:

$$
\forall_{i \in\{1 . . n\}} \forall_{x_{i} \in X_{i}} \exists_{x_{1} \in X_{1}, \ldots, x_{i-1} \in X_{i-1}, x_{i+1} \in X_{i+1}, \ldots, x_{n} \in X_{n}}\left(x_{1}, x_{2}, \ldots, x_{n}\right) \in R
$$

where variables are used to denote their domains.

In general, pair-wise AC is a weaker condition than GAC. For example, consider the following constraint: 


$$
\begin{array}{r}
(X, Y, Z) \text { in } \quad(0,1,1), \\
(1,0,1), \\
(1,1,0)]
\end{array}
$$

After the assignment $\mathrm{X}=1, \mathrm{Y}=1$, and $\mathrm{Z}=1$, the constraint is not GAC but it is still pair-wise AC.

When a table constraint is generated, tuples of variables and values in the form $\left(X_{1}, \ldots, X_{n}\right)$ are all transformed into the form $\mathrm{t}\left(X_{1}, \ldots, X_{n}\right)$ that takes less memory to store and is easier to manipulate.

\section{$2.2 \quad$ CLP(FD)}

CLP(FD) [8] is a constraint language that enhances Prolog with built-ins for specifying domain variables, constraints, and strategies for assigning values to variables (called labeling). The unification operator is enhanced to deal with domain variables. For two domain variables $X$ and $Y$, after unification $X=Y$ the elements that are not in both domains are removed and the two variables become aliases.

The following built-ins are used in the implementation of table constraints:

- $X$ in $D$ : restricts $X$ to take on a value from $D$, where $D$ is a set of integers.

- $X$ notin $D$ : forbids $X$ to take on any value from $D$.

- $f d \_d o m(X, D): D$ is the list of integers in the domain of $X$.

- fd_disjoint $(X, Y)$ : The domains of $X$ and $Y$ are disjoint.

- fd_set_false $(X, E)$ : excludes integer $E$ from the domain of $X$. It is equivalent to $X \# \backslash=E$ but more efficient.

These built-ins are available in B-Prolog. Similar built-ins are also available in other CLP(FD) systems or can be implemented using other primitives.

\subsection{Action rules and events}

The AR (Action Rules) language is designed to facilitate the specification of event-driven functionality needed by applications such as constraint propagators and graphical user interfaces where interactions of multiple entities are essential [16]. It was originally implemented in B-Prolog and now has been introduced into other Prolog systems [4].

An action rule takes the following form:

$$
\text { Agent }, \text { Condition, }\{\text { Event }\}=>\text { Action }
$$

where Agent is an atomic formula that represents a pattern for agents, Condition is a conjunction of conditions on the agents, Event is a non-empty disjunction of patterns for events that can activate the agents, and Action is a sequence of arbitrary subgoals. An action rule degenerates into a commitment rule if Event 


\section{Binary Constraints}

Given a binary table constraint $(X, Y)$ in $R$, we build a hashtable Hxy for supports of values of $\mathrm{X}^{2}$ For each value Ex in the domain of X, there exists an entry $(\mathrm{Ex}, \mathrm{Sx})$ in Hxy where $\mathrm{Sx}$ is a finite-domain variable that represents Ex's set of supports in the domain of Y. If Ex has only one support, then Sx is the support itself. Similarly, we also build a hashtable Hyx for supports of values of Y. After $(\mathrm{X}, \mathrm{Y})$ in $\mathrm{R}$ is posted, it is made $\mathrm{AC}$ by excluding all unsupported values from the domains of $\mathrm{X}$ and $\mathrm{Y}$. In the following, Hxy and Hyx are called support tables.

Consider, for example, the following constraint:

$$
(X, Y) \text { in }[(1,2),(2,1),(3,4),(3,5),(4,4)]
$$

The hashtable Hxy contains

$$
(1,2),(2,1),(3, \mathrm{~S} 3:[4,5]),(4,4)
$$

and the hashtable Hyx contains

$$
(1,2),(2,1),(4, \mathrm{~S} 4:[3,4]),(5,3)
$$

where S3: $[4,5]$ and $S 4:[3,4]$ are two finite-domain variables. After the constraint is posted, X's domain becomes $[1,2,3,4]$ and Y's domain becomes $[1,2,4,5]$.

The two hashtables Hxy and Hyx are essentially two tries [6]. Each trie requires, in the worst case, $\mathrm{O}(|\mathrm{X}| \times|\mathrm{Y}|)$ space. This representation is compact because of the indexing effect and the use of bit vectors for domain variables. As will be shown below, supports are never updated during search. Therefore, the domain variables used to represent supports never post any event.

For a binary constraint over $(\mathrm{X}, \mathrm{Y})$, we generate propagators to watch ins and dom_any events on $\mathrm{X}$ and $\mathrm{Y}$. The propagation is very straightforward. When $\mathrm{X}$ is bound to an integer, Y's domain is reduced to retain only those elements that are supported by $\mathrm{X}$. Whenever a value is excluded from the domain of $\mathrm{X}$, the supports of the value in the domain of $Y$ are examined and those values that are no longer supported by $\mathrm{X}$ are excluded from the domain of $\mathrm{Y}$.

The propagator watch_ins (X, Y, Hxy), defined below, watches ins $(X)$ events.

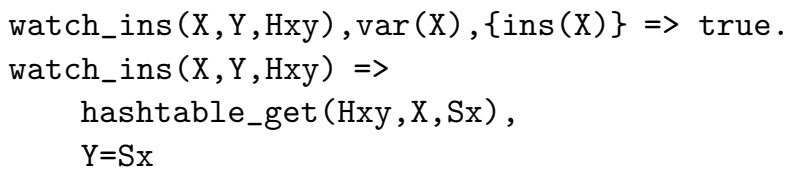

The propagator is suspended as long as $\mathrm{X}$ is a variable. The second rule is applied after $\mathrm{X}$ becomes ground, which unifies $\mathrm{Y}$ with the set of supports of $\mathrm{X}$.

The propagator watch_dom (X,Y, Hxy , Hyx ), defined in Figure 1, watches dom_any events on $\mathrm{X}$.

\footnotetext{
${ }^{2}$ Hashtables are not available in ISO-Prolog. The built-in hashtable_get (H,K,Val) in B-Prolog retrieves from the table $\mathrm{H}$ the value $\mathrm{Val}$ with the key $\mathrm{K}$. In the real implementation, a hashtable talored to tuples is used when the table is sparse or contain negative integers, and a structure is used otherwise.
} 


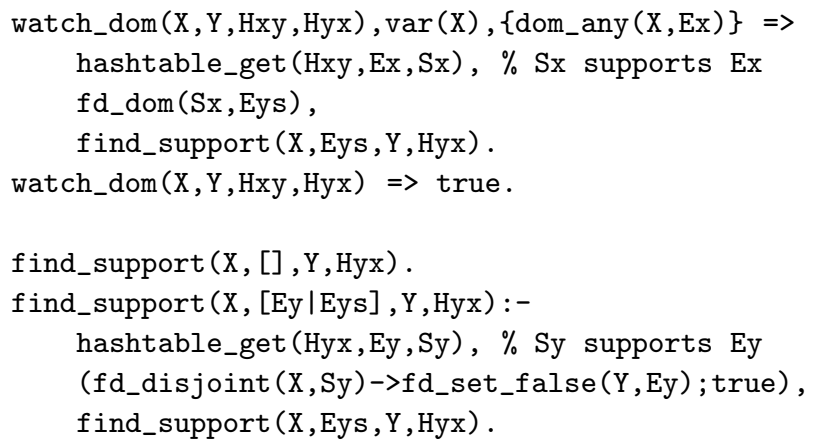

Fig. 1. The propagator that watches dom_any events.

Whenever a value Ex is excluded from the domain of X, Ex's set of supports $\mathrm{Sx}$ is retrieved from Hxy. The predicate find_support examines every element in $\mathrm{Sx}$, and excludes it from the domain of $\mathrm{Y}$ if it is no longer supported by $\mathrm{X}$. In the real implementation, find_support is encoded in $\mathrm{C}$ which uses bit-wise operations to iterate through the elements of Sx.

\section{Pair-wise AC for n-ary Constraints}

Given an n-ary table constraint Vars in R, we build two hashtables Hxy and Hyx for each pair of variables $\mathrm{X}$ and $\mathrm{Y}$ in Vars, and generate propagators to watch ins and dom_any events. In this way, pair-wise AC is maintained.

Since pair-wise AC does not guarantee GAC, an n-ary constraint needs to be checked after it becomes ground. Let HashR be the hashtable representation of the table R. This final check is described as follows:

final_check (HashR, Vars), n_vars_gt $(1,0),\{$ ins $(\operatorname{Vars})\} \Rightarrow$ true.

final_check $($ HashR, Vars $) \Rightarrow$ hashtable_get (HashR, Vars,_).

The condition n_vars_gt $(1,0)$ means that the last argument (namely Vars) has more than 0 variables. ${ }^{3}$ The subgoal is suspended while at least one of the variables in Vars is free. After all the variables are instantiated, hashtable_get checks if the tuple Vars is included in HashR.

\footnotetext{
${ }^{3}$ In general the built-in n_vars_gt $(m, n)$ in B-Prolog means that the number of variables in the last $\mathrm{m}$ arguments of the head is greater than $\mathrm{n}$, where both $\mathrm{m}$ and $\mathrm{n}$ are integer constants. Notice that the arguments are not passed to the built-in. The system always fetches those arguments from the current frame. This built-in is well used in constraint propagators to change the action when the number of variables in the constraint reaches a certain threshold.
} 


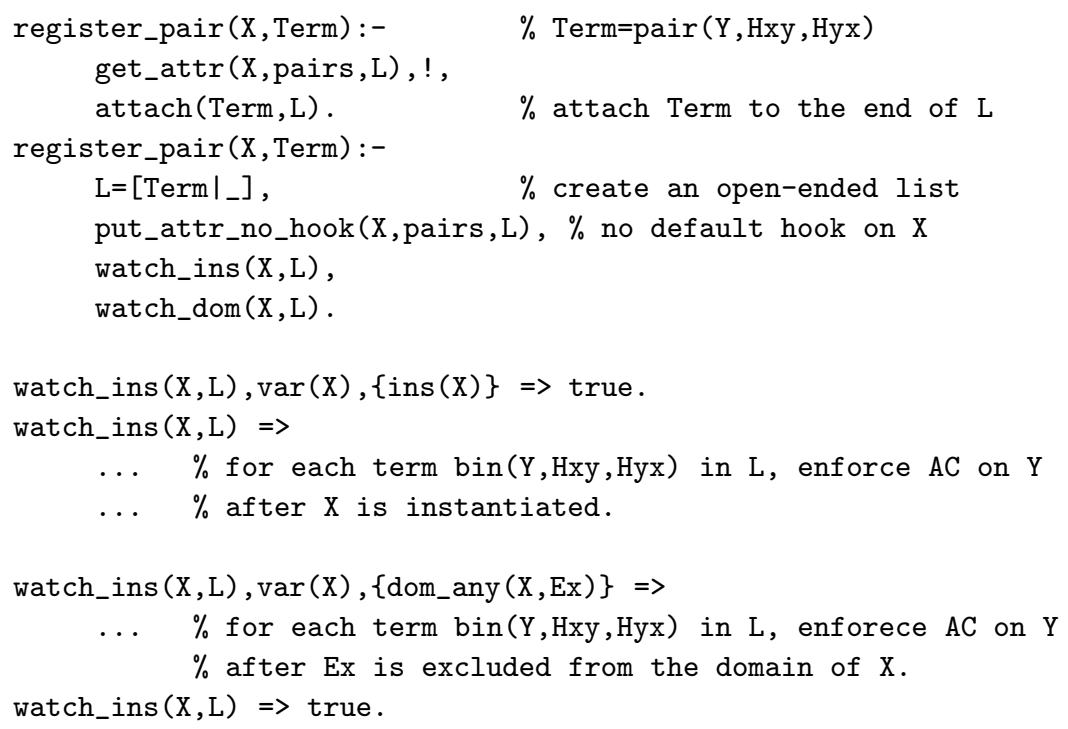

Fig. 2. The registration procedure.

\section{Improvements}

In the encoding described above, two propagators are used for each variable in a pair of variables, one watching ins and the other watching dom_any events on the variable. When a variable is involved in $n$ pairs, $2 \times n$ propagators are generated. One implementation technique for speeding-up propagation in CLP(FD) is to combine the propagators that watch the same event and take similar actions. ${ }^{4}$ This technique can be used to speed-up propagation for table constraints too.

For a pair of variables (X,Y), let Hxy and Hyx be the two support tables. The term pair ( $Y, H x y, H y x)$ is created and registered onto $X$ under the attribute name pairs. If the attribute pairs does not exist yet, the attribute is created and two propagators are generated; if the attribute already exists, then the term is attached to the end of the attribute value, which is an incomplete list with an open end. Figure 2 gives part of the registration procedure. Similarly, the term bin ( $\mathrm{X}, \mathrm{Hyx}, \mathrm{Hxy})$ needs to be registered onto Y.

The registration procedure is further improved as follows. For a pair $(X, Y)$, if the support tables Hxy and Hyx represent the Cartesian product of the domains of the variables, it is unnecessary to do the registration at all because every value is guaranteed a support no matter how the variables are instantiated.

Moreover, if a pair has been registered already, we merge the old support tables with the new ones. Let pair ( $Y, H x y, H y x)$ be the term to be registered onto $\mathrm{X}$, and pair $(\mathrm{Y}, \mathrm{OldHxy}, \mathrm{OldHyx})$ be a term that has been already registered

\footnotetext{
${ }^{4}$ This technique is implemented in B-Prolog for constraints such as disequality constraints over two variables.
} 
on X. We construct two new support tables NewHxy and NewHyx where NewHxy is the intersection of 0ldHxy and Hxy, and NewHyx is the intersection of OldHyx and Hyx. Then we use the built-in setarg/3 to replace 0ldHxy with NewHxy and OldHyx with NewHyx. This improvement allows inter-constraints sharing.

\section{Early Checking}

As shown above, pair-wise AC is weaker than GAC in terms of pruning power. The propagators for maintaining pair-wise $\mathrm{AC}$ resort to a final check to ensure that a constraint is indeed supported when it becomes ground. To remedy the weak pruning power of pair-wise AC, we can advance this final check to a point when the constraint still contain variables. An early check ensures that every value in the domain of every variable has a supporting tuple. We consider early checking for positive constraints, and similar ideas can be applied to negative constraints too.

There are two possible approaches to checking a constraint: One is to iterate through the values of the domains of the remaining variables in the constraint and, for each combination, we check if it is included in the table; the other is to iterate through the tuples in the table. Since the number of tuples in a given table is normally significantly smaller than the possible combinations of domain values when the number of variables is large, we follow the later approach.

To make it fast to iterate through the tuples in a table, we convert the table to a trie such that common prefixes of the tuples need not be examined more than once for each traversal. We only use one trie per table. The tuples are indexed on the first argument first, then second, and so on. The following defines a propagator that maintains GAC when variables are instantiated.

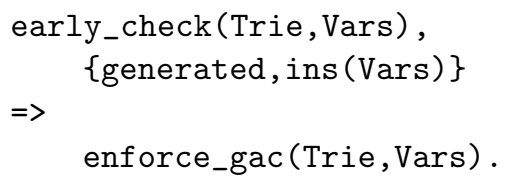

The predicate enforce_gac(Trie,Vars) is also called when the propagator is first created. It first walks through the trie to record all the values that are supported, and then it examines each value in the domain of each variable in Vars and excludes it from the domain if it has no supporting tuple.

Since enforce_gac does not respond to domain value exclusions, pair-wise $\mathrm{AC}$ is still weaker than GAC even with this early checking. To always enforce GAC, we could call ensure_gac whenever a change occurs to the domain of any variable.

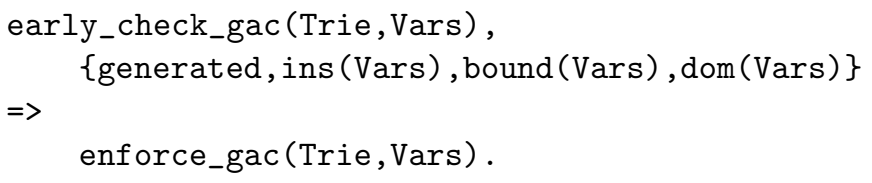


In addition to the generated and ins(Vars) events, this rule also watches bound (Vars) and dom(Vars) events. Recall that bound(Vars) is posted whenever a bound of the domain of any variable in Vars is changed and dom(Vars) is posted whenever an inner value is excluded from the domain of any variable. The two events bound(Vars) and dom(Vars) can be equivalently encoded as dom_any (Vars).

Since ensure_gac is expensive, calling it on every change may not pay off. One compromise is to enforce GAC on domain value exclusions only when the constraint contains a certain number of variables. The following gives the refined propagator for early checking.

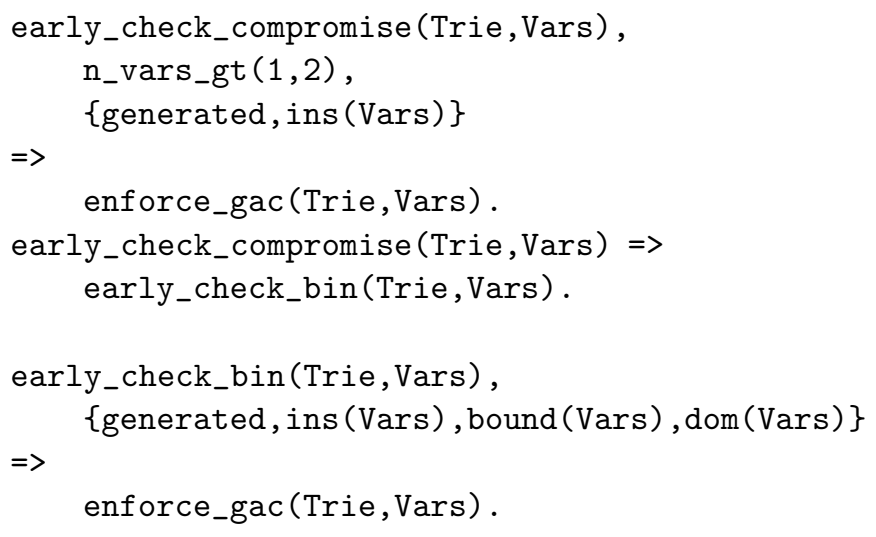

Once the number of variables contained in the constraint is 2 or less (the condition n_vars_gt $(1,2)$ fails), the propagator is replaced with early_check_bin which watches all changes to the domains of the variables.

Recall that the propagators for maintaining pair-wise AC already watch dom_any events. One may ask why we need to create a propagator to watch dom events here when the constraint becomes binary. The answer is that the support tables used in maintaining pair-wise AC are binary projections and they are never reduced while variables are instantiated. Consider the following example:

$$
\begin{array}{r}
(X, Y, Z) \text { in }[(0,1,1), \\
(0,2,2), \\
(0,3,3), \\
(1,1,2), \\
(1,2,3), \\
(1,3,1)]
\end{array}
$$

The support table Hyz from $Y$ to $Z$ contains the following entries: $(1, S 1:[1,2])$, $(2, \mathrm{~S} 2:[2,3])$, and $(3, \mathrm{~S} 3:[1,3])$. When $\mathrm{X}$ is bound to 0 , the support table should be reduced to contain $(1,1),(2,2)$, and $(3,3)$. After that, when a value, say 2 , is excluded from the domain of $Y$, the support 2 should be excluded from the domain of Z. Nevertheless, because our solver does not reduce support tables, this effect couldn't be achieved without calling enforce_gac(Trie,Vars). 


\section{$7 \quad$ Experimental Results}

The two built-ins, in/2 and notin/2, in B-Prolog have been extended to allow positive and negative table constraints. ${ }^{5}$ For positive constraints, pair-wise $\mathrm{AC}$ is used with early checking, which maintains GAC when constraints become ternary. For negative constraints, pair-wise AC is used with forward checking, which maintains GAC only when constraints become unary. For negative constraints, support tables are constructed without complementing given relations.

Thanks to the availability of action rules, the extension was implemented with relative ease. The extension contains about 300 lines of code in Prolog (and action rules) and 1000 lines of code in C, most of which are for preprocessing tables.

We compared pair-wise $\mathrm{AC}$ with and without early checking on a selected set of benchmarks used for the N-ARY-EXT category in the CSP solver competitions. ${ }^{6}$ The problem instances were translated from XML into Prolog format. The first 5 instances in each of seven selected problem classes were chosen. Each of the problem instances contains at least one positive constraint. Each instance was given a time limit of 500 seconds and no memory limit was imposed. The labeling strategy ffc (first-fail, breaking tie by selecting a most constrained variable) was used in all the runs. The machine used was a Pentium $3.0 \mathrm{GHz}$ with $1 \mathrm{~GB}$ of RAM running Windows XP.

Table 1 shows the CPU times. In each row, the first column gives the name of a problem instance, the second column gives the maximum arity of the constraints in the instance, and each of the remaining columns gives the CPU time taken by each of the four different settings: PAC maintains pair-wise AC without early checking; PAC+ET1 triggers early checking after constraints become binary; PAC+ET2 triggers early checking after constraints become ternary; and GAC maintains GAC all the time as is done in early_check_gac shown above. Both PAC+ET1 and PAC+ET2 trigger early checking on ins events. PAC solved only 16 instances, PAC+ET1 solved 28 instances, and PAC+ET2 and GAC each solved 30 instances. In general, PAC alone is too weak, but it turned out to be the fastest on the bdd benchmarks. There is no remarkable difference between $\mathrm{PAC}+\mathrm{ET} 2$ and GAC for most of the instances, and PAC+ET2 is faster than GAC on some for the instances such as crossword_m1c_lex_vg10_11. Four of the five instances of renault were not solved. Profiling the runs indicated that these instances were very memory demanding and most of the execution time was spent on garbage collection.

Table 2 shows the number of backtracks in each run. For those runs that were terminated by time-out events, the numbers were also recorded. For instances that contain only Boolean variables (bdd and jnh), there is no difference among different settings for early checking since no dom or dom_any event can occur on

\footnotetext{
5 Table constraints are supported in version 7.3 and up.

${ }^{6}$ http://www.cril.univ-artois.fr/ lecoutre/research/benchmarks/benchmarks.html
} 
Table 1. Comparison on CPU time (seconds).

\begin{tabular}{|c|c|c|c|c|c|}
\hline Problem instance & MAX-ARITY & $\overline{\mathrm{PAC}}$ & $\mathrm{PAC}+\mathrm{ET} 1$ & $\mathrm{PAC}+\mathrm{ET} 2$ & $\mathrm{GAC}$ \\
\hline bdd_21_133_18_78_10 & 18 & 1.50 & 2.70 & 2.82 & 2.67 \\
\hline bdd_21_133_18_78_11 & 18 & 9.25 & 31.00 & 31.00 & 31.00 \\
\hline bdd_21_133_18_78_12 & 18 & 7.85 & 24.00 & 24.00 & 23.00 \\
\hline bdd_21_133_18_78_13 & 18 & 9.32 & 69.00 & 69.00 & 69.00 \\
\hline bdd_21_133_18_78_14 & 18 & 11.00 & 33.00 & 33.00 & 33.00 \\
\hline crossword_m1c_lex_vg10_11 & 11 & $>500$ & 2.96 & 2.96 & 12.00 \\
\hline crossword_m1c_lex_vg10_12 & 12 & $>500$ & 0.61 & 0.59 & 2.61 \\
\hline crossword_m1c_lex_vg11_12 & 12 & $>500$ & 0.32 & 0.32 & 0.89 \\
\hline crossword_m1c_lex_vg11_13 & 13 & $>500$ & 0.12 & 0.12 & 0.25 \\
\hline crossword_m1c_lex_vg11_15 & 15 & 0.31 & 0.47 & 0.47 & 0.12 \\
\hline jnh01 & 14 & 0.15 & 0.15 & 0.15 & 0.15 \\
\hline jnh02 & 10 & 0.16 & 0.16 & 0.16 & 0.15 \\
\hline jnh04 & 11 & 0.20 & 0.20 & 0.20 & 0.20 \\
\hline jnh05 & 11 & 0.15 & 0.15 & 0.15 & 0.15 \\
\hline jnh06 & 11 & 0.11 & 0.11 & 0.11 & 0.94 \\
\hline rand_10_20_10_5_10000_0 & 10 & $>500$ & 1.42 & 1.42 & 1.43 \\
\hline rand_10_20_10_5_10000_10 & 10 & $>500$ & 1.29 & 1.28 & 1.29 \\
\hline rand_10_20_10_5_10000_11 & 10 & $>500$ & 1.87 & 1.82 & 2.46 \\
\hline rand_10_20_10_5_10000_12 & 10 & $>500$ & 1.31 & 1.31 & 1.87 \\
\hline rand_10_20_10_5_10000_13 & 10 & $>500$ & 1.37 & 1.37 & 1.92 \\
\hline renault_mgd & 10 & 2.50 & 2.57 & 2.54 & 2.57 \\
\hline renault_mod_0 & 10 & $>500$ & $>500$ & $>500$ & $>500$ \\
\hline renault_mod_10 & 10 & $>500$ & $>500$ & $>500$ & $>500$ \\
\hline renault_mod_11 & 10 & $>500$ & $>500$ & $>500$ & $>500$ \\
\hline renault_mod_12 & 10 & $>500$ & $>500$ & $>500$ & $>500$ \\
\hline ssa_0432_003 & 5 & 1.21 & 0.14 & 0.14 & 0.15 \\
\hline ssa_2670_130 & 5 & $>500$ & $>500$ & $>500$ & $>500$ \\
\hline ssa_2670_141 & 4 & 0.000 & 0.000 & 0.000 & 0.000 \\
\hline ssa_6288_047 & 6 & 0.40 & 0.40 & 0.40 & 0.40 \\
\hline ssa_7552_038 & 6 & 0.47 & 0.47 & 0.31 & 0.31 \\
\hline tsp_20_142 & 3 & $>500$ & $>500$ & 81.00 & 81.00 \\
\hline tsp_20_190 & 3 & $>500$ & 286.00 & 11.00 & 11.00 \\
\hline tsp_20_193 & 3 & $>500$ & $>500$ & 36.00 & 36.00 \\
\hline tsp_20_1 & 3 & $>500$ & 57.00 & 0.95 & 0.95 \\
\hline tsp_20_29 & 3 & $>500$ & 2.31 & 0.29 & 0.29 \\
\hline
\end{tabular}

Boolean variables. For instances that contain no constraint with more than 3 variables (tsp), there is no difference between PAC+ET2 and GAC.

We didn't directly compare our solver with other solvers for table constraints. The top ranked solvers, such as mddc, MDG, Mistral, and Abscon solved all the selected instances under a time limit of 1800 seconds. ${ }^{7}$ We have to mention that the Windows-XP PC we used is probably slower than the Linux server used in the competition and our solver does not employ any restart strategy. Even under the same condition, it would be unfair to compare a CLP(FD) solver with a solver implemented directly in $\mathrm{C}$ or $\mathrm{C}++$ because operations such dereferencing, tagging, and untagging incur measurable overhead in CLP(FD).

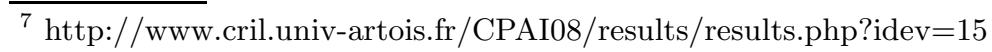


Table 2. Comparison on backtracks.

\begin{tabular}{|c|r|r|r|r|}
\hline Problem instance & PAC & PAC+ET1 & PAC+ET2 & GAC \\
\hline bdd_21_133_18_78_10 & 0 & 0 & 0 & 0 \\
bdd_21_133_18_78_11 & 532563 & 9734 & 9734 & 9734 \\
bdd_21_133_18_78_12 & 321883 & 13438 & 13438 & 13438 \\
bdd_21_133_18_78_13 & 535481 & 11154 & 11154 & 11154 \\
bdd_21_133_18_78_14 & 552313 & 10235 & 10235 & 10235 \\
crossword_m1c_lex_vg10_11 & 91408432 & 675 & 675 & 193 \\
crossword_m1c_lex_vg10_12 & 11401605 & 140 & 140 & 57 \\
crossword_m1c_lex_vg11_12 & 10616917 & 61 & 61 & 22 \\
crossword_m1c_lex_vg11_13 & 2100191 & 19 & 19 & 12 \\
crossword_m1c_lex_vg11_15 & 0 & 0 & 0 & 0 \\
jnh01 & 50 & 50 & 50 & 50 \\
jnh02 & 8 & 8 & 8 & 8 \\
jnh04 & 1611 & 1611 & 1611 & 1611 \\
jnh05 & 41 & 41 & 41 & 41 \\
jnh06 & 826 & 826 & 826 & 826 \\
rand_10_20_10_5_10000_0 & $>268435455$ & 1010 & 1010 & 999 \\
rand_10_20_10_5_10000_10 & $>268435455$ & 1000 & 1000 & 999 \\
rand_10_20_10_5_10000_11 & 263869300 & 1003 & 1003 & 999 \\
rand_10_20_10_5_10000_12 & $>268435455$ & 1002 & 1002 & 997 \\
rand_10_20_10_5_10000_13 & $>268435455$ & 1882 & 1882 & 998 \\
renault_mgd & 13 & 0 & 0 & 0 \\
renault_mod_0 & $>268435455$ & 17614672 & 13761572 & 20704205 \\
renault_mod_10 & 80724776 & 9859177 & 4931019 & 2200586 \\
renault_mod_11 & $>268435455$ & 8099239 & 3215349 & 1818967 \\
renault_mod_12 & 251771657 & 9082875 & 6301406 & 2128999 \\
ssa_0432_003 & 318288 & 10126 & 10126 & 10126 \\
ssa_2670_130 & 72698460 & 23508987 & 23994708 & 24034014 \\
ssa_2670_141 & 7 & 0 & 0 & 0 \\
ssa_6288_047 & 23 & 23 & 23 & 23 \\
ssa_7552_038 & 476 & 38 & 38 & 38 \\
tsp_20_142 & 17326326 & 207966 & 15036 & 15036 \\
tsp_20_190 & 25459227 & 366646 & 5750 & 5750 \\
tsp_20_193 & 17622012 & 207826 & 2814 & 2814 \\
tsp_20_1 & 21302231 & 65937 & 229 & 229 \\
tsp_20_29 & 29304024 & 2723 & 31 & 31 \\
\hline \hline
\end{tabular}

\section{Related and Further Work}

The key operation used in GAC algorithms is to find a support tuple for a value $y$ in the domain of a variable $Y$ after a value $x$ has been excluded from the domain of a related variable $X(X \neq Y)$ [2]. Significant efforts have been made to speedup this operation by skipping irrelevant tuples that can never been supports for a value $[3,6,10]$. Indexing is an effective technique. The trie data structure [6] indexes tuples such that tuples that have the same prefix share nodes in the trie. In order to facilitate propagating changes originated at every variable in an $\mathrm{n}$-ary constraint, the solver reported in $[6]$ needs to build $n$ tries, one for each variable. An MDD (multi-valued decision diagram) [3] is more effective than a trie in the sense that tuples that have the same suffix also share nodes. The solver mddc-solv based on MDD was ranked top in the N-ARY-EXT category in the third CSP solver competition. 
Our encoding of binary constraints is similar to the trie encoding. The difference is that the children (leaves) of each interior node are represented as a finite-domain variable rather than a list or an array. This representation fits CLP(FD) since bit-vectors are used in the representation of finite domains and bits in bit vectors are not tagged individually. Any data structure that requires tagging and untagging would incur considerable overhead.

It is well known that any n-ary constraint can be binarized by using a dual representation (i.e., treating each constraint as a variable) or introducing hidden variables for constraints [1]. Experiments have been done to compare various binarization schemes [14]. Our previous solver [17], like the early version of the Mistral solver [7]), introduces a new finite-domain variable for each n-ary constraint and encodes each tuple in the table as an integer. The main problem with that solver was that newly introduced variables could have very bigger domains and the solver could be flooded with events from these domains.

No previous work has been reported on introducing table constraints into CLP(FD). The case constraint in SICStus Prolog is used to implement the built-in table/2. Similar built-ins such as fd_relation/2 in GNU-Prolog and tuples_in/2 in SWI-Prolog have been implemented, but no detail of the implementation is published. None of these CLP(FD) systems directly supports negative table constraints.

In our solver, supports of values are not updated during search. This makes it possible for constraints to share tables and also renders it unnecessary to trail or copy supports of values. The drawback is that the support tables created for maintaining pair-wise $\mathrm{AC}$ for an n-ary constraint cannot be used to enforce $\mathrm{AC}$ when the constraint becomes binary. The early-checking propagators in our solver need to use the trie from the original table to enforce AC. Also the operation fd_disjoint does not become as cheap as it is supposed to be because the domain that represents supports of a value never shrinks. Recently, a new approach has been proposed that solves n-ary CSPs by reducing tables $[10,15]$. It is worthwhile to investigate if this approach can be integrated into our approach.

Further work needs to be done to investigate when and how early checking should be performed. Our solver does not do any early checking on negative constraints. Further investigation should cover negative constraints as well.

\section{Conclusion}

We have presented an encoding for table constraints in CLP(FD) based on pairwise AC. In the encoding, the supports of each value are represented as a finitedomain variable, and action rules are used to propagate value exclusions. The encoding is compact and requires no new problem variables. To remedy the weak pruning power of pair-wise AC, we proposed integrating pair-wise AC with early checking. Our experimental results showed that such an integration is effective. Our approach differs from the major GAC algorithms in that it is based on pair- 
wise $\mathrm{AC}$ and is fine grained. More work remains to be done concerning when and how early checking should be performed.

\section{References}

1. Fahiem Bacchus, Xinguang Chen, Peter van Beek, and Toby Walsh. Binary vs. non-binary constraints. Artif. Intell., 140(1/2):1-37, 2002.

2. Christian Bessière and Jean-Charles Régin. Arc consistency for general constraint networks: Preliminary results. In IJCAI (1), pages 398-404, 1997.

3. Kenil C. K. Cheng and Roland H. C. Yap. Maintaining generalized arc consistency on ad hoc r-ary constraints. In CP, pages 509-523, 2008.

4. Bart Demoen and Phuong-Lan Nguyen. Two WAM implementations of action rules. In ICLP, pages 621-635, 2008.

5. Eugene C. Freuder. Synthesizing constraint expressions. Commun. ACM, 21(11):958-966, 1978.

6. Ian P. Gent, Christopher Jefferson, Ian Miguel, and Peter Nightingale. Data structures for generalised arc consistency for extensional constraints. In $A A A I$, pages 191-197, 2007.

7. Emmanuel Hebrard. Mistral, a constraint satisfaction library. In M.R.C. van Dongen, Christophe Lecoutre, and Olivier Roussel, editors, Proceedings of the Second International CSP Solver Competition, pages 35-42, 2008.

8. Pascal Van Hentenryck. Constraint Satisfaction in Logic Programming. MIT Press, 1989.

9. George Katsirelos and Toby Walsh. A compression algorithm for large arity extensional constraints. In CP, pages 379-393, 2007.

10. Christophe Lecoutre. Optimization of simple tabular reduction for table constraints. In $C P$, pages 128-143, 2008.

11. Christophe Lecoutre and Vion Julien. Enforcing arc consistency using bitwise operations. Constraint Programming Letters, 2:21-35, 2008.

12. Alan K. Mackworth. On reading sketch maps. In IJCAI, pages 598-606, 1977.

13. Roger Mohr and Thomas C. Henderson. Arc and path consistency revisited. Artificial Intelligence, 28:225-233, 1986.

14. Kostas Stergiou and Nikos Samaras. Binary encodings of non-binary constraint satisfaction problems: Algorithms and experimental results. J. Artif. Intell. Res. (JAIR), 24:641-684, 2005.

15. Julian R. Ullmann. Partition search for non-binary constraint satisfaction. Inf. Sci., 177(18):3639-3678, 2007.

16. Neng-Fa Zhou. Programming finite-domain constraint propagators in action rules. Theory and Practice of Logic Programming (TPLP), 6(5):483-508, 2006.

17. Neng-Fa Zhou. BPSOLVER 2008. In M.R.C. van Dongen, Christophe Lecoutre, and Olivier Roussel, editors, Proceedings of the Third International CSP Solver Competition, pages 83-90, 2008. 